\title{
Parameterized T-Norm and Co-Norm based Intuitionistic Fuzzy Optimization Technique and its Application
}

\author{
Samir Dey \\ Deapartment of Methematics, \\ Asansol Engineering College \\ Vivekananda Sarani \\ Asansol-713305,India
}

\author{
Tapan Kumar Roy \\ Department of Mathematics, \\ Indian Institute of Engineering \\ Science and Technology \\ Botanic Gargen,Howrah-711103,India
}

\begin{abstract}
Real world engineering problems are usually designed by the presence of many conflicting objectives. In this paper, an approach is developed to solve multi-objective structural design using parameterized t-norms and t-co-norms based intuitionistic fuzzy optimization technique. Here binary $\mathrm{t}-$ norms, t-conorms are extended in the form of n-ary t-norms and t-co-norms and their basic properties are discussed with some special cases. In this paper we have considered a multi objective structural optimization model with weight and deflection as objectives and stress as constraint function. Here design variables are considered as cross sectional area of bars. This classical truss optimization example is presented here in to demonstrate the efficiency of our proposed optimization approach. Numerical example is given here to illustrate this structural model through this approximation method.
\end{abstract}

\section{Keywords}

Intuitionistic Fuzzy Set, T-norms, T-conorms, Structural Optimization.

\section{INTRODUCTION}

Structural design optimization is a challenging research topic nowadays. The challenge is to design structure with light weight and with minimum cost. It has been seen that multi objectives conflict with each other, and optimizing the problem considering a single objective can result an unacceptable results with respect to the other objectives. A reasonable solution to a multi-objective problem is a set of solutions, each of which satisfies the objectives without being dominated by any other solution. There are two general approaches for multiple-objective optimization problem. One of this is , make individual objective functions by combining all objective functions into a single composite function or move all objective but one to the constraint set. In the former case, determination of a single objective can be made by utility theory or weighted sum method, where weights or utility functions are dependent on the decision-maker's preferences. Sometimes, it can be very difficult to accurately select these weights. In the latter case, as a constraining value must be established for each of these former objectives there is a problem to move objectives to the constraint set. Again this can be arbitrary. So in both cases, a set of solutions in exchange of single solution would return by optimization method for examination of trade-offs. For this reason, decision-makers often choose a set of good solutions considering the multiple objectives. The second general approach is finding an entire Pareto optimal solution set. A Pareto optimal set is nothing but a set of solutions that are non dominated with respect to each other. A Pareto optimal set of solution is one, when we go from any Pareto optimal solution to another Pareto optimal solution, there always at least one objective function improves with sacrifice of at least one other. Since the final solution of the decision-maker is always a trade-off when considering real-life problems Pareto optimal solution sets are often preferred.

It has been seen that numerous engineering design problem need to deal with imprecise data, manufacturing error or uncertainty of the environment during the design process. fuzzy as well as intuitionistic fuzzy optimization in case of structural engineering not only helps the engineers in their design and analysis of systems but also leads to significant advances and new discoveries in fuzzy optimization theory and technique. This fuzzy set theory was first introduced by Zadeh [4]. As an extension Intuitionistic fuzzy set theory was first introduced by Atanassov [3].When an imprecise information cannot be expressed by means of conventional fuzzy set, Intuitionistic Fuzzy set play an important role. In intuitionistic fuzzy (IF) set we usually consider degree of acceptance, degree of non membership and hesitancy function whereas we consider only membership function in fuzzy set. A few research works has been done on intuitionistic fuzzy optimization in the field of structural optimization. Dey et al.[2] used intuitionistic fuzzy technique to optimize single objective two bar truss structural model. Dey et al.[9] used multi-objective intuitionistic optimization technique in their paper on three bar truss structural model. This is the first time a parameterized intuitionistic multi-objective nonlinear programming is introduced in this paper with an application in structural design.

An important concept in fuzzy as well as intuitionistic fuzzy set theory are triangular norms and conforms which are nothing but a generalized intersection and union of fuzzy sets. Alsina et al.[6] introduced the t-norm in fuzzy set theory and suggested that the t-norms could be used for the intersection of fuzzy sets. G.Deschrijver et al.[7] introduced the concept of intuitionistic fuzzy t-norm and t-co norm to investigate the theorems for similar representation of aggregated $t$-norm and t-conorm

As per our best of knowledge, this is the first time parameterized t-norms and $\mathrm{t}$ - co-norm based intuitionistic fuzzy optimization programming technique is being used to solve multi-objective structural model in this paper. In the test problem we have considered three-bar planar truss subjected to a single load condition where the objective functions are weight of the truss and deflection of loaded joint and the design variables are the cross-sections of bars with the constraints as stresses in members.

The remainder of this paper is organized in the following way. In section 2structural optimization model is discussed. In section 3, mathematics Prerequisites is discussed with. extended n-ary t-norms and t-co-norms and calculation of 
some of special cases. In section 4, we discuss about weighted fuzzy aggregation. In section 5 , we proposed the technique to solve a multi-objective non-linear programming problem using extended parameterized t-norms and t-co-norm based intuitionistic fuzzy optimization. In section 6, multi-objective structural model is solved using extended parameterized tnorms and t-co-norm based intuitionistic fuzzy optimization. Numerical illustration of structural model of three bar truss and comparison of results by using different extended weighted t-norms and t-co-norm are discussed in section 7.Finally we draw conclusions in section 8 .

\section{MULTI-OBJECTIVE STRUCTURAL MODEL}

In the design problem of the structure i.e lightest weight of the structure and minimum deflection of the loaded joint that satisfies all stress constraints in members of the structure .In truss structure system, the basic parameters (including allowable stress ,etc) are known and the optimization's target is that identify the optimal bar truss cross-section area so that the structure is of the smallest total weight with minimum nodes displacement in a given load conditions .

The multi-objective structural model can be expressed as

Minimize $W T(A)$

Minimize $\delta(A)$

subject to $\sigma(A) \leq[\sigma]$

$A^{\min } \leq A \leq A^{\max }$

Where $A=\left[A_{1}, A_{2}, \ldots A_{n}\right]^{T}$ are the design variables for the cross section, $\mathrm{n}$ is the group number of design variables for the cross section bar, $W T(A)=\sum_{i=1}^{n} \rho_{i} A_{i} L_{i}$ is the total weight of the structure, $\delta(A)$ is the deflection of the loaded joint, where $L_{i}, A_{i}$ and $\rho_{i}$ are the bar length ,cross section area and density of the $i^{\text {th }}$ group bars respectively. $\sigma(A)$ is the stress constraint and $[\sigma]$ is allowable stress of the group bars under various conditions, $A^{\min }$ and $A^{\text {max }}$ are the lower and upper bounds of cross section area A respectively.

\section{MATHEMATICAL PRELIMINARIES}

\subsection{Fuzzy Set}

Let $X$ denotes a universal set. Then the fuzzy subset $A$ in $X$ is a subset of order pairs $\tilde{A}=\left\{\left(x, \mu_{\tilde{A}}(x)\right): x \in X\right\}$ where $\mu_{\tilde{A}}: X \rightarrow[0,1]$ is called the membership function which assigns a real number $\mu_{\tilde{A}}(x)$ in the interval $[0,1]$ to each element $x \in X$. A is non fuzzy and $\mu_{\tilde{A}}(x)$ is identical to the characteristic function of crisp set. It is clear that the range of membership function is a subset of non-negative real numbers.

\subsection{Intuitionistic Fuzzy Set}

Let $X=\left\{x_{1}, x_{2}, \ldots, x_{n}\right\}$ be a finite universal set. An intuitionistic fuzzy set (IFS) set $A^{i}$ in the sense of Atanassove [3] is given by equation $\tilde{A}^{i}=\left\{<X, \mu_{\tilde{A}^{i}}(x), v_{\tilde{A}^{i}}(x)>\mid x_{i} \in X\right\}$ where the function $\quad \mu_{\tilde{A}^{i}}\left(x^{i}\right): X \rightarrow[0,1]$;

$x_{i} \in X \rightarrow \mu_{\tilde{A}^{i}}\left(x_{i}\right) \in[0,1]$ and $\quad v_{\tilde{A}^{i}}\left(x^{i}\right): X \rightarrow[0,1]$

$x_{i} \in X \rightarrow v_{A^{i}}\left(x_{i}\right) \in[0,1]$ define the degree of membership and degree of non-membership of an element $x_{i} \in X$ to the set $\tilde{A}^{i} \subseteq X$, such that they satisfy the condition $0 \leq \mu_{\tilde{A}^{i}}\left(x_{i}\right)+v_{\tilde{A}^{i}}\left(x_{i}\right) \leq 1, \forall x_{i} \in X$. For each IFS $\tilde{A}^{i}$ in $X$ the amount $\Pi_{\tilde{A}^{i}}\left(x_{i}\right)=1-\left(\mu_{\tilde{A}^{i}}\left(x^{i}\right)+v_{\tilde{A}^{i}}\left(x^{i}\right)\right)$ is called the degree of uncertainty (or hesitation ) associated with the membership of elements $x_{i} \in X$ in $\tilde{A}^{i}$ we call it intuitionistic fuzzy index of $\tilde{A}^{i}$ with respect of an element $x_{i} \in X$.

\section{$3.3 \alpha$-Level set or $\alpha$ - cut of a Fuzzy Set}

The $\alpha$-level set of a fuzzy set $A$ of $X$ is a crisp set $A_{\alpha}$ which contains all the elements of $X$ that have membership values greater than or equal to $\alpha$ i.e $A=\left\{x: \mu_{A}(x) \geq \alpha, x \in X, \alpha \in[0,1]\right\}$.

\section{4 $(\alpha, \beta)$ cut of a Intuitionistic Fuzzy Set}

A set of $(\alpha, \beta)$ - cut generated by IFS $\tilde{A}^{i}$ where $\alpha, \beta \in[0,1]$ are fixed number such that $\alpha+\beta \leq 1$ is denoted by

$\tilde{A}_{\alpha, \beta}^{i}=\left\{\begin{array}{l}<x, \mu_{\tilde{A}^{i}}(x), v_{\tilde{A}^{i}}(x)>: x \in X, \\ \mu_{\tilde{A}^{i}}(x) \geq \alpha, v_{\tilde{A}^{i}}(x) \leq \beta, \alpha, \beta \in[0,1]\end{array}\right\}$

and defined as the crisp set of element $x$ which belong to $\tilde{A}^{i}$ at least to the degree $\alpha$ and which belong to $\tilde{A}^{i}$ at most to the degree $\beta$.

\subsection{Triangular Norm (T-Norm)}

$T:[0,1] \times[0,1] \rightarrow[0,1]$ is said to be t-norm if it satisfies the following properties

i) $T(a, b)=T(b, a) \forall a, b \in[0,1] \quad$ (commutativity)

ii) $T(T(a, b), c)=T(a, T(b, c)) \forall a, b, c \in[0,1]$ (Associativity)

iii) $T(a, b) \leq T(a, c)$ with $b \leq c \quad \forall a, b, c \in[0,1]$

(Monotonocity)

iv) $T(0,0)=0, T(1,1)=1$;

v) $T(a, 1)=a \forall a \in[0,1]$

(Identity)

\subsection{Extended n-ary Triangular Norm}

For the purpose of operations of multiple fuzzy sets ,it is useful to define the notation of multidimensional t-norms. Let $[0,1]^{n}$ be a n-dimensional cube and $\left(x_{1}, x_{2}, \ldots ., x_{n}\right)$ and $\left(z_{1}, z_{2}, \ldots \ldots, z_{n}\right) \in[0,1]^{n}$.A mapping $T:[0,1]^{n} \rightarrow[0,1]$ is called $\mathrm{n}$-dimensional $\mathrm{t}$-norm if it satisfies the following properties.

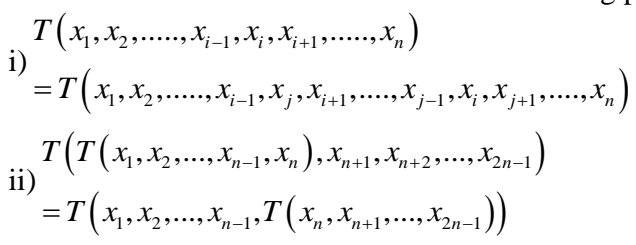


iii) For $\left(x_{1}, x_{2}, \ldots, x_{n-1}, x_{n}\right) \leq\left(z_{1}, z_{2}, \ldots, z_{n-1}, z_{n}\right) \Rightarrow$

$T\left(x_{1}, x_{2}, \ldots, x_{n-1}, x_{n}\right) \leq T\left(z_{1}, z_{2}, \ldots, z_{n-1}, z_{n}\right)$ with $x_{i}=z_{i}$ for some

$i$ and $x_{i} \leq z_{i}$ for some $i=1,2, \ldots ., n$

iv) $T(0,0, \ldots ., 0)=0, T(1,1, \ldots, 1)=1$

v) $T\left(1,1, \ldots, x_{i}, \ldots, 1\right)=x_{i}$

\subsection{Properties of Extended n -ary Triang- ular Norm (T-Norm)}

Due to associative law it is easy to extend a triangular norm $T$ into $\mathrm{n}$ arguments the $\mathrm{n}$-ary operation $T_{n}$ on $[0,1]$ satisfies the following properties

i) $T_{n}\left(x_{1}, x_{2}, \ldots, x_{n}\right)=T_{n}\left(x_{\sigma_{1}}, x_{\sigma_{2}}, \ldots, x_{\sigma_{n}}\right)$ where $\quad \sigma$ is $\quad$ a permutation of $\{1,2, \ldots \ldots, n\} \quad$ (Commutativity)

ii) $T_{n}\left(x_{1}, x_{2}, \ldots, x_{n}\right)=T_{i+1}\left(x_{1}, x_{2}, \ldots, x_{i}, T_{n-i}\left(x_{i+1}, . ., x_{j}, \ldots, x_{n}\right)\right)$

$=T_{n-j+1}\left(T\left(x_{1}, x_{2}, \ldots, x_{j}\right), x_{j+1}, \ldots, x_{n}\right)$

iii) $\left(\forall i \in N_{n}\right)\left(x_{i} \leq x_{i}^{\prime}\right) \Rightarrow$

$T_{n}\left(x_{1}, x_{2}, \ldots, x_{n}\right) \leq T_{n}\left(x_{1}^{\prime}, x_{2}^{\prime}, \ldots, x_{n}^{\prime}\right)$

(monotonocity)

$T_{n}\left(x_{1}, x_{2}, \ldots, x_{i-1}, 1, x_{i+1}, \ldots, x_{n}\right)$

iv) $=T\left(x_{1}, x_{2}, \ldots, x_{i-1}, x_{i+1}, . ., x_{j}, \ldots, x_{n}\right)$

(Identity Law)

A t-norm $T_{n}$ is said to be continuous if $T$ is continuous function on[0,1].From the above, we may call $T_{n}$ an extension of triangular norm .In the sequel we omit number of argument $\mathrm{n}$ and simply write $T$ of the class of mapping generated by triangular norm $T$.

\subsection{Triangular Conorm (T-Conorm)}

$S:[0,1] \times[0,1] \rightarrow[0,1]$ is said to be t-conorm if it satisfies the following properties

i) $S(a, b)=S(b, a) \forall a, b \in[0,1]$

(commutativity)

ii) $S(S(a, b), c)=S(a, S(b, c)) \forall a, b, c \in[0,1]$

(Associativity)

iii) $S(a, b) \leq S(a, c)$ with $\quad b \leq c \forall a, b, c \in[0,1]$

(Monotonocity)

iv) $S(0,0)=0, S(1,1)=1$;

v) $S(a, 0)=a \forall a \in[0,1]$ (Identity)

\subsection{Extended n-ary Triangular Conorm (T-Conorm)}

For the purpose of operations of multiple fuzzy sets ,it is useful to define the notation of multidimensional t-norms. Let

$[0,1]^{n}$ be a n-dimensional cube and $\left(x_{1}, x_{2}, \ldots, x_{n}\right)$ and $\left(z_{1}, z_{2}, \ldots, z_{n}\right) \in[0,1]^{n}$.A mapping $S:[0,1]^{n} \rightarrow[0,1]$ is called ndimensional t-norm if it satisfies the following properties.

$$
\text { i) } \begin{aligned}
& S\left(x_{1}, x_{2}, \ldots, x_{i-1}, x_{i}, x_{i+1}, \ldots, x_{n}\right) \\
&= S\left(x_{1}, x_{2}, \ldots, x_{i-1}, x_{j}, x_{i+1}, \ldots, x_{j-1}, x_{i}, x_{j+1}, \ldots, x_{n}\right)
\end{aligned}
$$

ii) $S\left(S\left(x_{1}, x_{2}, \ldots, x_{n-1}, x_{n}\right), x_{n+1}, x_{n+2}, \ldots, x_{2 n-1}\right)$

$=S\left(x_{1}, x_{2}, \ldots, x_{n-1}, S\left(x_{n}, x_{n+1}, \ldots, x_{2 n-1}\right)\right)$

iii) For $\left(x_{1}, x_{2}, \ldots, x_{n-1}, x_{n}\right) \leq\left(z_{1}, z_{2}, \ldots, z_{n-1}, z_{n}\right) \Rightarrow$

$S\left(x_{1}, x_{2}, \ldots, x_{n-1}, x_{n}\right) \leq S\left(z_{1}, z_{2}, \ldots, z_{n-1}, z_{n}\right)$ with $x_{i}=z_{i}$ for some

$i$ and $x_{i} \leq z_{i}$ for some $i=1,2, \ldots, n$

iv) $S(0,0, \ldots, 0)=0, S(1,1, \ldots, 1)=1$

v) $S\left(0,0, \ldots, x_{i}, \ldots, 0\right)=x_{i}$

\subsection{Properties of Extended n-ary Triang-} ular Conorm (T-Norm)

Due to associative law it is easy to extend a triangular norm $S$ into $\mathrm{n}$ arguments the n-ary operation $S_{n}$ on $[0,1]$ satisfies the following properties

i) $S_{n}\left(x_{1}, x_{2}, \ldots, x_{n}\right)=S_{n}\left(x_{\sigma_{1}}, x_{\sigma_{2}}, \ldots, x_{\sigma_{n}}\right)$ where $\quad \sigma$ is $\quad$ a permutation of $\{1,2, \ldots, n\}$

(Commutativity)

ii) $S_{n}\left(x_{1}, x_{2}, \ldots, x_{n}\right)=S_{i+1}\left(x_{1}, x_{2}, \ldots, x_{i}, S_{n-i}\left(x_{i+1}, \ldots, x_{j}, \ldots, x_{n}\right)\right)$

$=S_{n-j+1}\left(S\left(x_{1}, x_{2}, \ldots, x_{j}\right), x_{j+1}, \ldots, x_{n}\right)$

iii) $\left(\forall i \in N_{n}\right)\left(x_{i} \leq x_{i}^{\prime}\right)$

iii)

$\Rightarrow S_{n}\left(x_{1}, x_{2}, \ldots, x_{n}\right) \leq S_{n}\left(x_{1}^{\prime}, x_{2}^{\prime}, \ldots, x_{n}^{\prime}\right)$

(monotonocity)

$S_{n}\left(x_{1}, x_{2}, \ldots, x_{i-1}, 0, x_{i+1} \ldots, x_{n}\right)$

iv) $=S\left(x_{1}, x_{2}, \ldots, x_{i-1}, x_{i+1}, . ., x_{j}, \ldots, x_{n}\right)$

(Identity Law)

A t-norm $S_{n}$ is said to be continuous if $S$ is continuous function on $[0,1]$.From the above, we may call $S_{n}$ an extension of triangular norm .In the sequel we omit number of argument $\mathrm{n}$ and simply write $S$ of the class of mapping generated by triangular norm $S$.

\subsection{Four Basic T-norm and T-conorm and their Generalization with Weight}

Let $\tilde{A}_{j}^{i}=\left\{\begin{array}{l}<x_{j}, \mu_{\tilde{A}_{j}^{i}}\left(x_{j}\right), v_{\tilde{A}_{j}^{i}}\left(x_{j}\right)>: x_{j} \in X, \\ \mu_{\tilde{A}_{j}^{i}}\left(x_{j}\right) \geq \alpha, v_{\tilde{A}_{j}^{i}}\left(x_{j}\right) \leq \beta, \alpha, \beta \in[0,1]\end{array}\right\}$

be $\mathrm{n}$ intuitionistic fuzzy set for $j=1,2, \ldots ., n$.

i)Minimum t-norm and maximum t-conorm

The intuitionistic fuzzy minimum t-norm and maximum t-conorm can be defined as

$$
\begin{aligned}
& T_{M}\left(\mu_{1}\left(x_{1}\right), \mu_{2}\left(x_{2}\right), \ldots \ldots, \mu_{n}\left(x_{n}\right)\right) \\
& =\min \left\{\mu_{1}\left(x_{1}\right), \mu_{2}\left(x_{2}\right), \ldots \ldots, \mu_{n}\left(x_{n}\right)\right\} \text { and } \\
& S_{M}\left(v_{1}\left(x_{1}\right), v_{2}\left(x_{2}\right), \ldots \ldots, v_{n}\left(x_{n}\right)\right) \\
& =\max \left\{v_{1}\left(x_{1}\right), v_{2}\left(x_{2}\right), \ldots \ldots, v_{n}\left(x_{n}\right)\right\}
\end{aligned}
$$

Similarly $\mathrm{n}$-ary intuitionistic fuzzy minimum t-norm and maximum t-co-norm

with weight can be defined as 
$T_{M}^{w}\left(w_{1}, \mu_{1}\left(x_{1}\right) ; w_{2}, \mu_{2}\left(x_{2}\right) ; \ldots . ; w_{n}, \mu_{n}\left(x_{n}\right)\right)$

$=\min \left\{w_{1} \mu_{1}\left(x_{1}\right) ; w_{2} \mu_{2}\left(x_{2}\right) ; \ldots . ; w_{n} \mu_{n}\left(x_{n}\right)\right\}$

$S_{M}^{w}\left(w_{1}, v_{1}\left(x_{1}\right) ; w_{2}, v_{2}\left(x_{2}\right) ; \ldots . . ; w_{n}, v_{n}\left(x_{n}\right)\right)$

$=\max \left\{w_{1} v_{1}\left(x_{1}\right) ; w_{2} v_{2}\left(x_{2}\right) ; \ldots . ; w_{n} v_{n}\left(x_{n}\right)\right\}$

ii) Probabilistic t-norm and t-conorm

The intuitionistic fuzzy probabilistic t-norm and t-co-norm can be defined as

$$
\begin{aligned}
& T_{P}\left(\mu_{1}\left(x_{1}\right), \mu_{2}\left(x_{2}\right), \ldots . ., \mu_{n}\left(x_{n}\right)\right)=\prod_{i=1}^{n}\left(\mu_{i}\left(x_{i}\right)\right) \text { and } \\
& S_{P}\left(v_{1}\left(x_{1}\right), v_{2}\left(x_{2}\right), \ldots . ., v_{n}\left(x_{n}\right)\right)=1-\prod_{i=1}^{n}\left(1-v_{i}\left(x_{i}\right)\right)
\end{aligned}
$$

Similarly n-ary intuitionistic fuzzy probabilistic t-norm and tco-norm with weight can be defined as

$T_{P}^{w}\left(w_{1}, \mu_{1}\left(x_{1}\right) ; w_{2}, \mu_{2}\left(x_{2}\right) ; \ldots . ; w_{n}, \mu_{n}\left(x_{n}\right)\right)=\prod_{i=1}^{n}\left(\mu_{i}\left(x_{i}\right)\right)^{w_{i}}$ and

$S_{P}^{w}\left(w_{1}, v_{1}\left(x_{1}\right) ; w_{2}, v_{2}\left(x_{2}\right) ; \ldots . . ; w_{n}, v_{n}\left(x_{n}\right)\right)=1-\prod_{i=1}^{n}\left(1-v_{i}\left(x_{i}\right)\right)^{w_{i}}$

iii)Lukasewicz t-norm and t-conorm

The intuitionistic fuzzy Lukasewicz t-norm and t-co-norm can be defined as

$$
\begin{aligned}
& T_{L}\left(\mu_{1}\left(x_{1}\right), \mu_{2}\left(x_{2}\right), \ldots . ., \mu_{n}\left(x_{n}\right)\right)=\max \left(\sum_{i=1}^{n} \mu_{i}\left(x_{i}\right)-(n-1), 0\right) \\
& \text { and } S_{L}\left(v_{1}\left(x_{1}\right), v_{2}\left(x_{2}\right), \ldots . ., v_{n}\left(x_{n}\right)\right)=\min \left\{1, \sum_{i=1}^{n} v_{i}\left(x_{i}\right)\right\}
\end{aligned}
$$

Similarly n-ary intuitionistic fuzzy Lukasewicz t-norm and tco-normwith weight can be defined as

$$
T_{L}^{w}\left(\mu_{1}\left(x_{1}\right), \mu_{2}\left(x_{2}\right), \ldots . ., \mu_{n}\left(x_{n}\right)\right)=\max \left(\sum_{i=1}^{n} w_{i} \mu_{i}\left(x_{i}\right)-(n-1), 0\right)
$$
and

$S_{L}^{w}\left(v_{1}\left(x_{1}\right), v_{2}\left(x_{2}\right), \ldots \ldots, v_{n}\left(x_{n}\right)\right)=\min \left\{1, \sum_{i=1}^{n} w_{i} v_{i}\left(x_{i}\right)\right\}$

iv)Weber (or Drastic Product ) t-norm and t-conorm

The intuitionistic fuzzy Weber (or Drastic Product ) t-norm and t-co-norm can be defined as

$$
\begin{aligned}
& T_{D}\left(\mu_{1}\left(x_{1}\right), \mu_{2}\left(x_{2}\right), \ldots \ldots, \mu_{n}\left(x_{n}\right)\right) \\
& =\left\{\begin{array}{c}
\min \left(\mu_{i}\left(x_{i}\right)\right) \text { if } \max \left(\mu_{i}\left(x_{i}\right)\right)=1 \\
0 \quad \text { otherwise }
\end{array}\right.
\end{aligned}
$$

And

$$
\begin{aligned}
& S_{D}\left(v_{1}\left(x_{1}\right), v_{2}\left(x_{2}\right), \ldots \ldots, v_{n}\left(x_{n}\right)\right) \\
& = \begin{cases}\max \left(v_{i}\left(x_{i}\right)\right) & \text { if } \min \left(v_{i}\left(x_{i}\right)\right)=0 \\
1 & \text { if } \min \left(v_{i}\left(x_{i}\right)\right)>0\end{cases}
\end{aligned}
$$

Similarly n-ary intuitionistic fuzzy Lukasewicz t-norm and tco-norm with weight can be defined as

$$
\begin{aligned}
& T_{D}^{w}\left(w_{1}, \mu_{1}\left(x_{1}\right) ; w_{2}, \mu_{2}\left(x_{2}\right) ; \ldots . . ; w_{n}, \mu_{n}\left(x_{n}\right)\right) \\
& =\left\{\begin{array}{cc}
\min \left(w_{i} \mu_{i}\left(x_{i}\right)\right) & \text { if } \max \left(w_{i} \mu_{i}\left(x_{i}\right)\right)=1 \text { and } \\
0 & \text { otherwise }
\end{array}\right. \\
& S_{D}^{w}\left(w_{1}, v_{1}\left(x_{1}\right) ; w_{2}, v_{2}\left(x_{2}\right) ; \ldots . ., w_{n}, v_{n}\left(x_{n}\right)\right) \\
& =\left\{\begin{array}{cc}
\max \left(w_{i} v_{i}\left(x_{i}\right)\right) & \text { if } \min \left(w_{i} v_{i}\left(x_{i}\right)\right)=0 \\
1 & \text { if } \min \left(w_{i} v_{i}\left(x_{i}\right)\right)>0
\end{array}\right.
\end{aligned}
$$

\subsection{Some Particular Classes of T-norms and T-co-norms}

A t-norm and t-co-norm is commutative order semi-group with unit element 1 on $[0,1]$ of real numbers, So the class of tnorm and t-co-norm is quite large. Two well-known class of tnorm and t-co-norm are discussed here.

Yager (1980) introduced the following classes of t-norms and t-co-normsas

$$
\begin{aligned}
& T_{\lambda}^{Y}\left(\mu_{1}\left(x_{1}\right), \mu_{2}\left(x_{2}\right)\right) \\
& =1-\min \left[1,\left\{\left(1-\mu_{1}\left(x_{1}\right)\right)^{\lambda}+\left(1-\mu_{2}\left(x_{2}\right)\right)^{\lambda}\right\}^{\left(\frac{1}{\lambda}\right)}\right] \forall \lambda \in[0, \infty)
\end{aligned}
$$

and

$$
\begin{aligned}
& S_{\lambda}^{Y}\left(v_{1}\left(x_{1}\right), v_{2}\left(x_{2}\right)\right) \\
& =\min \left[1,\left\{\left(v_{1}\left(x_{1}\right)\right)^{\lambda}+\left(v_{2}\left(x_{2}\right)\right)^{\lambda}\right\}^{\left(\frac{1}{\lambda}\right)}\right] \forall \lambda \in[0, \infty)
\end{aligned}
$$

Extended n-ary form of above t-norm is

$$
\begin{aligned}
& T_{\lambda}^{Y}\left(\mu_{1}\left(x_{1}\right), \mu_{1}\left(x_{2}\right), \ldots \ldots . . ., \mu_{n}\left(x_{n}\right)\right) \\
& =1-\min \left[1,\left\{\sum_{i=1}^{n}\left(1-\mu_{i}\left(x_{i}\right)\right)^{\lambda}\right\}^{\frac{1}{\lambda}}\right] \forall \lambda \in[0, \infty)
\end{aligned}
$$

and

$$
\begin{aligned}
& S_{\lambda}^{Y}\left(v_{1}\left(x_{1}\right), v_{1}\left(x_{2}\right), \ldots \ldots \ldots, v_{n}\left(x_{n}\right)\right) \\
& =\min \left[1,\left\{\sum_{i=1}^{n}\left(v_{i}\left(x_{i}\right)\right)^{\lambda}\right\}^{\frac{1}{\lambda}}\right] \forall \lambda \in[0, \infty)
\end{aligned}
$$

The extended form with different weights of the above tnorms and t-conorms are $T_{\lambda}^{Y}\left(w_{1}, \mu_{1}\left(x_{1}\right) ; w_{2}, \mu_{1}\left(x_{2}\right) ; \ldots ; w_{n}, \mu_{n}\left(x_{n}\right)\right)$ $=1-\min \left[1,\left\{\sum_{i=1}^{n}\left(1-w_{i} \mu_{i}\left(x_{i}\right)\right)^{\lambda}\right\}^{\frac{1}{\lambda}}\right] \forall \lambda \in[0, \infty)$ and 
$S_{\lambda}^{Y}\left(w_{1}, v_{1}\left(x_{1}\right) ; w_{2}, v_{1}\left(x_{2}\right) ; \ldots \ldots . . ; w_{n}, v_{n}\left(x_{n}\right)\right)$

$=\min \left[1,\left\{\sum_{i=1}^{n}\left(w_{i} v_{i}\left(x_{i}\right)\right)^{\lambda}\right\}^{\frac{1}{\lambda}}\right] \forall \lambda \in[0, \infty)$

It can be prove that

$\begin{aligned} & \lim _{\lambda \rightarrow \infty} T_{\lambda}^{Y}\left(\mu_{1}\left(x_{1}\right), \mu_{1}\left(x_{2}\right), \ldots \ldots \ldots, \mu_{n}\left(x_{n}\right)\right) \\ = & T_{M}\left(\mu_{1}\left(x_{1}\right), \mu_{1}\left(x_{2}\right), \ldots \ldots . ., \mu_{n}\left(x_{n}\right)\right) \quad \forall \lambda \in[0, \infty)\end{aligned}$

Proof of (Ia) is given in the paper Dey and Roy [10]

(III) $\begin{aligned} & \lim _{\lambda \rightarrow 0} T_{\lambda}^{Y}\left(\mu_{1}\left(x_{1}\right), \mu_{1}\left(x_{2}\right), \ldots \ldots \ldots, \mu_{n}\left(x_{n}\right)\right) \\ & =T_{D}\left(\mu_{1}\left(x_{1}\right), \mu_{1}\left(x_{2}\right), \ldots \ldots . ., \mu_{n}\left(x_{n}\right)\right) \quad \forall \lambda \in[0, \infty)\end{aligned}$

Proof of (IIa) is given in the paper Dey and Roy [10]

(Ib) $\begin{aligned} & \lim _{\lambda \rightarrow \infty} S_{\lambda}^{Y}\left(v_{1}\left(x_{1}\right), v_{1}\left(x_{2}\right), \ldots \ldots \ldots, v_{n}\left(x_{n}\right)\right) \\ = & S_{M}\left(v_{1}\left(x_{1}\right), v_{1}\left(x_{2}\right), \ldots \ldots . ., v_{n}\left(x_{n}\right)\right) \quad \forall \lambda \in[0, \infty)\end{aligned}$

(IIb) $\begin{aligned} & \lim _{\lambda \rightarrow 0} S_{\lambda}^{Y}\left(v_{1}\left(x_{1}\right), v_{1}\left(x_{2}\right), \ldots \ldots \ldots, v_{n}\left(x_{n}\right)\right) \\ & =S_{D}\left(v_{1}\left(x_{1}\right), v_{1}\left(x_{2}\right), \ldots \ldots . ., v_{n}\left(x_{n}\right)\right) \quad \forall \lambda \in[0, \infty)\end{aligned}$

Proof of (Ib)

Case I.

Let $v_{1}\left(x_{1}\right)=v_{1}\left(x_{2}\right)=\ldots \ldots . . .=v_{n}\left(x_{n}\right) \neq 1$

Then

$\lim _{\lambda \rightarrow \infty} S_{\lambda}^{Y}\left(v_{1}\left(x_{1}\right), v_{1}\left(x_{2}\right), \ldots \ldots ., v_{n}\left(x_{n}\right)\right)$

$=\lim _{\lambda \rightarrow \infty}\left(\min \left[1,\left\{\sum_{i=1}^{n}\left(v_{i}\left(x_{i}\right)\right)^{\lambda}\right\}^{\frac{1}{\lambda}}\right]\right)$

$=\left(\min \left[1,\left\{\lim _{\lambda \rightarrow \infty} \sum_{i=1}^{n}\left(v_{i}\left(x_{i}\right)\right)^{\lambda}\right\}^{\frac{1}{\lambda}}\right]\right)$

$=\left(\min \left[1,\left\{\lim _{\lambda \rightarrow \infty}\left(n^{\frac{1}{\lambda}}\left(v_{i}\left(x_{i}\right)\right)\right)\right\}\right]\right)$

$=v_{i}\left(x_{i}\right)$

$=\max \left\{v_{1}(x), v_{2}(x), \ldots \ldots, v_{n}(x)\right\}$

$=S_{M}\left\{v_{1}(x), v_{2}(x), \ldots \ldots, v_{n}(x)\right\}$.

Case II.

Let $v_{1}\left(x_{1}\right)=v_{1}\left(x_{2}\right)=\ldots \ldots . .=v_{n}\left(x_{n}\right)=1$

Then

$\lim _{\lambda \rightarrow \infty} S_{\lambda}^{Y}\left(v_{1}\left(x_{1}\right), v_{1}\left(x_{2}\right), \ldots \ldots . ., v_{n}\left(x_{n}\right)\right)$

$$
\begin{aligned}
& =\min \left\{1,\left(\sum_{i=1}^{n}\left(v_{i}\left(x_{i}\right)\right)^{\lambda}\right)^{\frac{1}{\lambda}}\right\} \\
& =\min \{1,1\} \\
& =\max \left(v_{1}\left(x_{1}\right), v_{1}\left(x_{2}\right), \ldots \ldots . ., v_{n}\left(x_{n}\right)\right) \\
& =S_{M}\left(v_{1}\left(x_{1}\right), v_{1}\left(x_{2}\right), \ldots \ldots . ., v_{n}\left(x_{n}\right)\right)
\end{aligned}
$$

Case III.

Let $v_{i}\left(x_{i}\right) \neq v_{j}\left(x_{j}\right)$ for $i, j=1,2, \ldots, n, i \neq j$. Without loss of generality let us consider

$v_{1}\left(x_{1}\right)>v_{1}\left(x_{2}\right)>\ldots \ldots . .>v_{n}\left(x_{n}\right)$

Let $P=\left(\sum_{i=1}^{n}\left(\left(v_{i}\left(x_{i}\right)\right)^{\lambda}\right)^{\frac{1}{\lambda}}\right)$

Then $\lim _{\lambda \rightarrow \infty} \ln P=\lim _{\lambda \rightarrow \infty} \ln \left(\sum_{i=1}^{n}\left(v_{i}\left(x_{i}\right)\right)^{\lambda}\right)^{\frac{1}{\lambda}}$

$=\lim _{\lambda \rightarrow \infty} \frac{\ln \sum_{i=1}^{n}\left(v_{i}\left(x_{i}\right)\right)^{\lambda}}{\lambda}\left[\frac{\infty}{\infty}\right.$ form $]$

$=\lim _{\lambda \rightarrow \infty} \frac{\sum_{i=1}^{n}\left(v_{i}\left(x_{i}\right)\right)^{\lambda} \ln v_{i}\left(x_{i}\right)}{\sum_{i=1}^{n}\left(v_{i}\left(x_{i}\right)\right)^{\lambda}}$

$=\lim _{\lambda \rightarrow \infty} \frac{\ln \left(v_{1}\left(x_{1}\right)\right)^{2}+\sum_{i=1}^{n-1}\left(\frac{v_{i+1}\left(x_{i+1}\right)}{v_{i}}\right)^{\lambda}}{\ln v_{1}\left(x_{1}\right)+\sum_{i=1}^{n-1}\left(\frac{v_{i+1}\left(x_{i+1}\right)}{v_{i}}\right)^{\lambda}}$

$=\ln v_{1}\left(x_{1}\right)$

$\lim _{\lambda \rightarrow \infty} P=v_{1}\left(x_{1}\right)$

$=\max \left\{v_{1}\left(x_{1}\right), v_{2}\left(x_{2}\right), \ldots \ldots, v_{n}\left(x_{n}\right)\right\}$

$=S_{M}\left(v_{1}\left(x_{1}\right), v_{1}\left(x_{2}\right), \ldots \ldots \ldots, v_{n}\left(x_{n}\right)\right)$

Proof of (IIb)

Case I.

Let

$v_{1}\left(x_{1}\right)=. .=v_{i-1}\left(x_{i-1}\right)=v_{i+1}\left(x_{i-1}\right) \ldots=v_{n}\left(x_{n}\right)=0$ and $v_{i}\left(x_{i}\right) \neq 0$

Then

$\lim _{\lambda \rightarrow 0} S_{\lambda}^{Y}\left(v_{1}\left(x_{1}\right), v_{1}\left(x_{2}\right), \ldots \ldots . ., v_{n}\left(x_{n}\right)\right)$

$=\lim _{\lambda \rightarrow 0} \min \left\{1,\left(\sum_{i=1}^{n}\left(v_{i}\left(x_{i}\right)\right)^{\lambda}\right)^{\frac{1}{\lambda}}\right\}$ 


$$
\begin{aligned}
& =\min \left\{1, \lim _{\lambda \rightarrow 0}\left(v_{i}\left(x_{i}\right)\right)\right\} \\
& =v_{i}\left(x_{i}\right) \\
& =\max \left\{v_{1}\left(x_{1}\right), v_{1}\left(x_{2}\right), \ldots \ldots . ., v_{n}\left(x_{n}\right)\right\} \\
& =S_{D}\left\{v_{1}\left(x_{1}\right), v_{1}\left(x_{2}\right), \ldots \ldots \ldots, v_{n}\left(x_{n}\right)\right\}
\end{aligned}
$$

\section{Case II.}

Let $v_{i}\left(x_{i}\right)=0$ for $i=1,2, \ldots \ldots \ldots, n$

Then

$$
\begin{aligned}
& \lim _{\lambda \rightarrow 0} S_{\lambda}^{Y}\left(v_{1}\left(x_{1}\right), v_{1}\left(x_{2}\right), \ldots \ldots . ., v_{n}\left(x_{n}\right)\right) \\
& =\lim _{\lambda \rightarrow 0} \min \left\{1,\left(\sum_{i=1}^{n}\left(v_{i}\left(x_{i}\right)\right)^{\lambda}\right)^{\frac{1}{\lambda}}\right\} \\
& =\min \{1,0\} \\
& =0 \\
& =\max \left\{v_{1}\left(x_{1}\right), v_{2}\left(x_{2}\right), \ldots \ldots ., v_{n}\left(x_{n}\right)\right\} \\
& =S_{D}\left(v_{1}\left(x_{1}\right), v_{1}\left(x_{2}\right), \ldots \ldots \ldots, v_{n}\left(x_{n}\right)\right)
\end{aligned}
$$

Hamacher (1978) introduced the following classes of tnorms and t-co-norms as

$$
\begin{aligned}
& T_{\lambda}^{H}\left(\mu_{1}\left(x_{1}\right), \mu_{2}\left(x_{2}\right)\right) \\
& =\frac{\mu_{1}\left(x_{1}\right) \mu_{2}\left(x_{2}\right)}{\lambda+(1-\lambda)\left\{1-\left(1-\mu_{1}\left(x_{1}\right)\right)\left(1-\mu_{2}\left(x_{2}\right)\right)\right\}} \forall \lambda \in[0, \infty) \\
& \text { and }=\frac{v_{1}\left(x_{1}\right)+v_{2}\left(x_{2}\right)-(2-\lambda) v_{1}\left(x_{1}\right) v_{2}\left(x_{2}\right)}{1-(1-\lambda) v_{1}\left(x_{1}\right) v_{2}\left(x_{2}\right)} \quad \lambda \geq 0
\end{aligned}
$$

Extended n-ary form of above t-norm is

$$
\begin{aligned}
& T_{\lambda}^{H}\left(\mu_{1}\left(x_{1}\right), \mu_{2}\left(x_{2}\right), \ldots \ldots, \mu_{n}\left(x_{n}\right)\right) \\
& =\frac{\prod_{i=1}^{n} \mu_{i}\left(x_{i}\right)}{\lambda+(1-\lambda)\left\{1-\prod_{i=1}^{n}\left(1-\mu_{i}\left(x_{i}\right)\right)\right\}} \forall \lambda \in[0, \infty)
\end{aligned}
$$

And

$$
\begin{aligned}
& S_{\lambda}^{H}\left(v_{1}\left(x_{1}\right), v_{1}\left(x_{2}\right), \ldots \ldots \ldots, v_{n}\left(x_{n}\right)\right) \\
& =\frac{\sum_{i=1}^{n} v_{i}\left(x_{i}\right)-(2-\lambda) \prod_{i=1}^{n} v_{i}\left(x_{i}\right)}{1-(1-\lambda) \prod_{i=1}^{n} v_{i}\left(x_{i}\right)} \lambda \geq 0
\end{aligned}
$$

The extended form with different weights of the above tnorms and t-conorms are $T_{\lambda}^{H}\left(w_{1}, \mu_{1}\left(x_{1}\right) ; w_{2}, \mu_{2}\left(x_{2}\right) ; \ldots ; w_{n}, \mu_{n}\left(x_{n}\right)\right)$

$=\frac{\prod_{i=1}^{n}\left(\mu_{i}\left(x_{i}\right)\right)^{w_{i}}}{\lambda+(1-\lambda)\left\{1-\prod_{i=1}^{n}\left(1-\mu_{i}\left(x_{i}\right)\right)^{w_{i}}\right\}} \forall \lambda \in[0, \infty)$

and

$$
\begin{aligned}
& S_{\lambda}^{H}\left(w_{1}, v_{1}\left(x_{1}\right) ; w_{1}, v_{1}\left(x_{2}\right) ; \ldots \ldots \ldots ; w_{n}, v_{n}\left(x_{n}\right)\right) \\
& =\frac{\sum_{i=1}^{n} w_{i} v_{i}\left(x_{i}\right)-(2-\lambda) \prod_{i=1}^{n}\left(v_{i}\left(x_{i}\right)\right)^{w_{i}}}{1-(1-\lambda) \prod_{i=1}^{n}\left(v_{i}\left(x_{i}\right)\right)^{w_{i}}} \lambda \geq 0
\end{aligned}
$$

It can be prove that

$$
\text { (Ia) } \begin{aligned}
& \lim _{\lambda \rightarrow 1} T_{\lambda}^{H}\left(\mu_{1}\left(x_{1}\right), \mu_{1}\left(x_{2}\right), \ldots \ldots \ldots, \mu_{n}\left(x_{n}\right)\right) \\
& =T_{P}\left(\mu_{1}\left(x_{1}\right), \mu_{1}\left(x_{2}\right), \ldots \ldots \ldots, \mu_{n}\left(x_{n}\right)\right) \quad \forall \lambda \in[0, \infty)
\end{aligned}
$$

Proof of (Ia) is given in the paper Dey and Roy [10]

$$
\begin{aligned}
& \lim _{\lambda \rightarrow \infty} T_{\lambda}^{H}\left(\mu_{1}\left(x_{1}\right), \mu_{1}\left(x_{2}\right), . ., \mu_{n}\left(x_{n}\right)\right) \\
= & T_{D}\left(\mu_{1}\left(x_{1}\right), \mu_{1}\left(x_{2}\right), \ldots, \mu_{n}\left(x_{n}\right)\right) \forall \lambda \in[0, \infty)
\end{aligned}
$$

Proof of (IIa) is given in the paper Dey and Roy [10]

$$
\begin{aligned}
& \lim _{\lambda \rightarrow 1} S_{\lambda}^{Y}\left(v_{1}\left(x_{1}\right), v_{1}\left(x_{2}\right), \ldots, v_{n}\left(x_{n}\right)\right) \\
& =S_{P}\left(v_{1}\left(x_{1}\right), v_{1}\left(x_{2}\right), \ldots, v_{n}\left(x_{n}\right)\right) \forall \lambda \in[0, \infty) \\
& \lim _{\lambda \rightarrow \infty} S_{\lambda}^{Y}\left(v_{1}\left(x_{1}\right), v_{1}\left(x_{2}\right), \ldots, v_{n}\left(x_{n}\right)\right) \\
& =S_{D}\left(v_{1}\left(x_{1}\right), v_{1}\left(x_{2}\right), \ldots, v_{n}\left(x_{n}\right)\right) \forall \lambda \in[0, \infty)
\end{aligned}
$$

Proof of (Ib)

$$
\begin{aligned}
& \lim _{\lambda \rightarrow 1} S_{\lambda}^{H}\left(v_{1}\left(x_{1}\right), v_{1}\left(x_{2}\right), \ldots \ldots \ldots, v_{n}\left(x_{n}\right)\right) \\
& =\lim _{\lambda \rightarrow 1} \frac{\sum_{i=1}^{n} v_{i}\left(x_{i}\right)+(2-\lambda) \prod_{i=1}^{n} v_{i}\left(x_{i}\right)}{1-(1-\lambda) \prod_{i=1}^{n} v_{i}\left(x_{i}\right)} \\
& =1-\prod_{i=1}^{n}\left(1-v_{i}\left(x_{i}\right)\right) \\
& =S_{P}\left(v_{1}\left(x_{1}\right), v_{1}\left(x_{2}\right), \ldots \ldots . ., v_{n}\left(x_{n}\right)\right)
\end{aligned}
$$

Proof of (IIb)

Case I.

Let $v_{1}\left(x_{1}\right)=v_{1}\left(x_{2}\right)=\ldots \ldots . .=v_{n}\left(x_{n}\right)=0$

Then

$$
\lim _{\lambda \rightarrow \infty} S_{\lambda}^{H}\left(v_{1}\left(x_{1}\right), v_{1}\left(x_{2}\right), \ldots \ldots \ldots, v_{n}\left(x_{n}\right)\right)
$$


$=\lim _{\lambda \rightarrow \infty} \frac{\sum_{i=1}^{n} v_{i}\left(x_{i}\right)+(2-\lambda) \prod_{i=1}^{n} v_{i}\left(x_{i}\right)}{1-(1-\lambda) \prod_{i=1}^{n} v_{i}\left(x_{i}\right)}$

$=0$

$=\max \left\{v_{1}\left(x_{1}\right), v_{2}\left(x_{2}\right), \ldots \ldots ., v_{n}\left(x_{n}\right)\right\}$

$=S_{D}\left(v_{1}\left(x_{1}\right), v_{1}\left(x_{2}\right), \ldots \ldots \ldots, v_{n}\left(x_{n}\right)\right)$

Case II.

Let $v_{1}\left(x_{1}\right)=v_{1}\left(x_{2}\right)=\ldots \ldots . . .=v_{n}\left(x_{n}\right) \neq 0$

Then

$\lim _{\lambda \rightarrow \infty} S_{\lambda}^{H}\left(v_{1}\left(x_{1}\right), v_{1}\left(x_{2}\right), \ldots \ldots \ldots, v_{n}\left(x_{n}\right)\right)$

$=\lim _{\lambda \rightarrow \infty} \frac{\sum_{i=1}^{n} v_{i}\left(x_{i}\right)+(2-\lambda) \prod_{i=1}^{n} v_{i}\left(x_{i}\right)}{1-(1-\lambda) \prod_{i=1}^{n} v_{i}\left(x_{i}\right)}$

$=1$

$=S_{D}\left(v_{1}\left(x_{1}\right), v_{1}\left(x_{2}\right), \ldots \ldots \ldots, v_{n}\left(x_{n}\right)\right)$

Hence the proof.

\section{WEIGHTED INTUITIONISTIC FUZZY AGGREGATION}

Weighted aggregation has been used quiet extensively especially in fuzzy decision making, where the weight are used to represent the relative importance and the negligence the decision maker attaches to different decision criterion (goals or constraints).Weighted aggregation of fuzzy sets by using t-norm has been considered by Yagar [5].He proposed to modify the membership function with the associated weight factors before the fuzzy aggregation. Xeshui Xu [8] presented intuitionistic fuzzy aggregation operator .The weighted aggregation is then the aggregation of the modified membership and non-membership functions and the general form of this idea is

$$
\begin{aligned}
& D_{1}(x, w)=T\left(I\left(\mu_{1}\left(x_{1}\right), w_{1}\right), I\left(\mu_{2}\left(x_{2}\right), w_{2}\right), \ldots, I\left(\mu_{k}\left(x_{k}\right), w_{k}\right)\right) \\
& D_{2}(x, w)=S\left(I\left(v_{1}\left(x_{1}\right), w_{1}\right), I\left(v_{2}\left(x_{2}\right), w_{2}\right), \ldots, I\left(v_{k}\left(x_{k}\right), w_{k}\right)\right)
\end{aligned}
$$

Where $w$ are vectors of weight factor $w_{i} \in[0,1] i=1,2, \ldots ., k$ associated with the aggregated membership function $\mu_{i}\left(x_{i}\right)$ and non-membership function $v_{i}\left(x_{i}\right)$.Here $\mathrm{T}$ is triangular norm and $\mathrm{S}$ is triangular conorm , $\mathrm{I}$ is a function of two variables that transforms the membership and nonmembership with $\sum_{i=1}^{k} w_{i}=1, w_{i} \geq 0$;
5. MATHEMATICAL ANALYSIS

5.1 Intuitionistic Fuzzy Non-linear

Programming Optimization with

Different Weighted T-norm and T-

conorm Operator to Solve MultiObjective Non-linear Programming

\section{Problem}

A multi-objective non-linear parametric intuitionistic programming (MONLP) Problem can be formulated as

Minimize $\left\{f_{1}(x), f_{2}(x), \ldots ., f_{p}(x)\right\}^{T}$

Subject to $g_{j}(x) \leq b_{j} ; \quad j=1,2, \ldots \ldots, m$

$x>0$

Following Zimmermann [11], we have presented a solution algorithm to solve the MONLP Problem by fuzzy optimization technique.

Step-1: Solve the MONLP (2) as a single objective non-linear programming problem $p$ th by taking one of the objective at a time and ignoring the others .These solutions are known as ideal solutions. Let $x^{i}$ be the respective optimal solution for the $i^{\text {th }}$ different objectives with same constraints and evaluate each objective values for all these $i^{\text {th }}$ optimal solutions.

Step-2: From the result of step -1 determine the corresponding values for every objective for each derived solutions. With the values of all objectives at each ideal solutions, pay-off matrix can be formulated as follows

$$
\begin{aligned}
& f_{1}(x) \quad f_{2}(x) \quad \ldots \ldots . . . \quad f_{p}(x)
\end{aligned}
$$

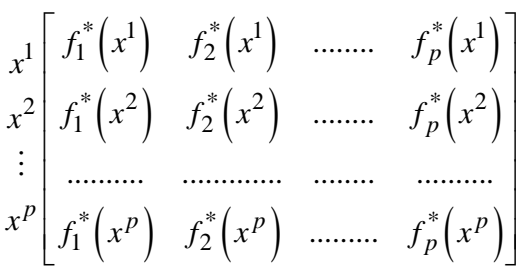

Here $x^{1}, x^{2}, \ldots \ldots, x^{p}$ are the ideal solution of the objectives $f_{1}(x), f_{2}(x), \ldots ., f_{p}(x)$ respectively.

Step-3: From the result of step 2 now we find lower bound (minimum) $L_{i}^{A C C}$

and upper bound (maximum) $U_{i}^{A C C}$ by using following rule $U_{i}^{A C C}=\max \left\{f_{i}\left(x^{p}\right)\right\}, L_{i}^{A C C}=\min \left\{f_{i}\left(x^{p}\right)\right\}$ where $1 \leq i \leq p$

.But in IFO The degree of non-membership (rejection) and the degree of membership (acceptance) are considered so that the sum of both value is less than one. To define the non membership of NLP problem let $U_{i}^{\operatorname{Re} j}$ and $L_{i}^{\operatorname{Re} j}$ be the upper bound and lower bound of objective function $f_{i}(x)$ where $L_{i}^{A C C} \leq L_{i}^{\operatorname{Re} j} \leq U_{i}^{\operatorname{Re} j} \leq U_{i}^{A C C}$.For objective function of minimization problem ,the upper bound for non-membership function (rejection) is always equals to that the upper bound of membership function (acceptance).One can take lower 
bound for non-membership function as follows $L_{i}^{\operatorname{Re} j}=L_{i}^{A c c}+\varepsilon_{i}$ where $0<\varepsilon_{i}<\left(U_{i}^{A c c}-L_{i}^{A c c}\right)$ based on the decision maker choice.

The initial intuitionistic fuzzy model with aspiration level of objectives becomes Find $\left\{x_{i}, i=1,2, \ldots ., p\right\}$

so as to satisfy $f_{i}(x) \leq i L_{i}^{A c c}$ with tolerance $P_{i}^{A c c}=\left(U_{i}^{A c c}-L_{i}^{A c c}\right)$ for the degree of acceptance for $i=1,2, \ldots \ldots, p . \quad f_{i}(x) \geq^{i} U_{i}^{\operatorname{Re} j}$ with tolerance $P_{i}^{A c c}=\left(U_{i}^{A c c}-L_{i}^{A c c}\right)$ for degree of rejection for $i=1,2, \ldots \ldots, p$.Define the membership (acceptance) and nonmembership (rejection) functions of above uncertain objectives as follows. For the $i^{\text {th }}, i=1,2, \ldots, p$ objectives functions the linear membership function $\mu_{i}\left(f_{i}(x)\right)$ and linear non-membership $v_{i}\left(f_{i}(x)\right)$ is defined as follows

$\mu_{i}\left(f_{i}(x)\right)=\left\{\begin{array}{cc}1 & \text { if } f_{i}(x) \leq L_{i}^{A c c} \\ \frac{e^{-T\left(\frac{f_{i}(x)-L_{i}^{A c c}}{U_{i}^{A c c}-L_{i}^{A c c}}\right)}-e^{-T}}{1-e^{-T}} & \text { if } L_{i}^{A c c} \leq f_{i}(x) \leq U_{i}^{A c c} \\ 0 & \text { if } \quad f_{i}(x) \geq U_{i}^{A c c}\end{array}\right.$

$v_{i}\left(f_{i}(x)\right)=\left\{\begin{array}{ccc}0 & \text { if } \quad f_{i}(x) \leq L_{i}^{\operatorname{Re} j} \\ \left(\frac{f_{i}(x)-L_{i}^{\operatorname{Re} j}}{U_{i}^{\operatorname{Re} j}-L_{i}^{\operatorname{Re} j}}\right)^{2} & \text { if } & L_{i}^{\operatorname{Re} j} \leq f_{i}(x) \leq U_{i}^{\operatorname{Re} j} \\ 1 & \text { if } \quad & f_{i}(x) \geq U_{i}^{\operatorname{Re} j}\end{array}\right.$

After determining the different membership functions for each of the objective functions, one can adopt following three type of decisions

i) According to the extension of the weighted intuitionistic min-max operator the MONLP (2)

can be formulated as

Maximize $\mu_{\tilde{D}_{1}}(x ; w)=$

$\operatorname{Maximize}\left(\operatorname{Minimum}\left\{w_{1} \mu_{1}\left(f_{1}(x)\right), \ldots, w_{p} \mu_{p}\left(f_{p}(x)\right)\right\}\right)$

Minimize $v_{\tilde{D}_{2}}(x ; w)$

$=\operatorname{Minimize}\left(\operatorname{Maximum}\left\{w_{1} v_{1}\left(f_{1}(x)\right), \ldots . ., w_{p} v_{p}\left(f_{p}(x)\right)\right\}\right)$

such that

$0 \leq \mu_{i}\left(f_{i}(x)\right)+v_{i}\left(f_{i}(x)\right) \leq 1 ;$ for $i=1,2, \ldots, p$.

$\mu_{i}\left(f_{i}(x)\right) \geq v_{i}\left(f_{i}(x)\right)$, for $i=1,2, \ldots, p$.

$\mu_{i}\left(f_{i}(x)\right) \in[0,1], v_{i}\left(f_{i}(x)\right) \in[0,1]$, for $i=1,2, \ldots, p$.

$g_{j}(x) \leq b_{j} ; j=1,2, \ldots ., m$. $x>0 ; \sum_{i=1}^{p} w_{i}=1 ; w_{i i} \in[0,1]$ for $i=1,2, \ldots, p$.

According to Angelov [1] the above problem can be formulated as

Maximize $(\alpha-\beta)$

$w_{i} \mu_{i}\left(f_{i}(x)\right) \geq \alpha ; w_{i} v_{i}\left(f_{i}(x)\right) \leq \beta ;$ for $i=1,2, . ., p$.

$g_{j}(x) \leq b_{j} ;$ for $j=1,2, \ldots, m$.

$x>0 ; 0 \leq \alpha+\beta \leq 1 ; \alpha \geq \beta ; \alpha, \beta \in[0,1]$

$x>0 ; \sum_{i=1}^{p} w_{i}=1 ; w_{i} \in[0,1]$

ii)According to the extension of the weighted intuitionistic Yager (1980) operatorthe MONLP (2) can be formulated as

Maximize $\mu_{\tilde{D}_{1}}(x ; w)$

$=$ Maximize $\left\{0,1-\sqrt[\lambda]{\sum_{i=1}^{p} w_{i}\left(1-\mu_{i}\left(f_{i}(x)\right)\right)}\right\} \lambda>0$

Minimize $v_{\tilde{D}_{2}}(x ; w)=$ Minimize $\left\{1, \lambda \sqrt{\sum_{i=1}^{p} w_{i} v_{i}\left(f_{i}(x)\right)}\right\} \lambda>0$,

Subject to the same constraint as (i)

iii)According to the extension of the weighted intuitionistic Hamecher (1978) operator the MONLP (2) can be formulated as

Maximize $\mu_{\tilde{D}_{1}}(x ; w)$

$=\operatorname{Max} \frac{\prod_{i=1}^{p}\left(\mu_{i}\left(f_{i}(x)\right)\right)^{w_{i}}}{\lambda+(1-\lambda)\left\{1-\prod_{i=1}^{p}\left(1-\mu_{i}\left(f_{i}(x)\right)\right)^{w_{i}}\right\}} \lambda \leq 2$

(5)

$$
\begin{aligned}
& \text { Minimize } v_{\tilde{D}_{2}}(x ; w) \\
& =\operatorname{Min} \frac{\sum_{i=1}^{p} w_{i}\left(v_{i}\left(f_{i}(x)\right)\right)-(2-\lambda) \prod_{i=1}^{p}\left(v_{i}\left(f_{i}(x)\right)\right)^{w_{i}}}{1-(1-\lambda) \prod_{i=1}^{p}\left(v_{i}\left(f_{i}(x)\right)\right)^{w_{i}}} \lambda \geq 0,
\end{aligned}
$$

Subject to the same constraint as (i)

Step-4: Solving any of the above two we will get the optimal solution of MONLP (2).

\subsection{Pareto Optimality Test}

A numerical test of Pareto optimality for $x^{*}$ can be performed by solving the following problem

Maximize $R=\sum_{i=1}^{p} \xi_{i}$

(6)

subject to

$f_{i}(x)+\xi_{i}=f_{i}\left(x^{*}\right) i=1,2, \ldots, p$.

$x \in X, \xi_{i} \geq 0 i=1,2, \ldots, p$. 
The optimal solution of (3), say $x^{* *}$ and $f_{i}\left(x^{* *}\right)$ are called strong Pareto optimal. Solution provided $\xi_{i}$ is very small, otherwise it is called weak Pareto-optimal Solution

\section{SOLUTION OF MULTI-OBJECTIVE STRUCTURAL OPTIMIZATION PROBLEM BY INTUITIONISTIC FUZZY OPTIMIZATION TECHNIQUE}

To solve the MOSOP (1) step 1 of 5.1is used. After that according to step 2 pay-off matrix is formulated

$$
\begin{aligned}
& W T(A) \quad \delta(A)
\end{aligned}
$$

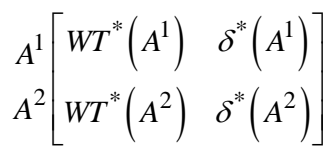

In next step following step 2 we calculate the bound of the objective $U_{W T}^{A c c}, L_{W T}^{A c c}$ and $U_{W T}^{\operatorname{Re} j}, L_{W T}^{\operatorname{Re} j}$ for weight function $W T(A)$, such that $\quad L_{W T}^{A c c}<W T(A)<U_{W T}^{A c c}$ and $L_{W T}^{\operatorname{Re} j}<W T(A)<U_{W T}^{\operatorname{Re} j}$ and $\quad U_{\delta}^{A c c}, L_{\delta}^{A c c} ; U_{\delta}^{\operatorname{Re} j}, L_{\delta}^{\operatorname{Re} j}$ for deflection $\quad \delta(A)$, such that $L_{\delta}^{A c c}<\delta(A)<U_{\delta}^{A c c}$ and $L_{\delta}^{\operatorname{Re} j}<\delta(A)<U_{\delta}^{\operatorname{Re} j}$ with the condition $U_{i}^{A c c}=U_{i}^{\operatorname{Re} j} ;$ $L_{i}^{\operatorname{Re} j}=L_{i}^{A c c}+\varepsilon_{i} \quad$ for $i=W T, \delta$ so $0<\varepsilon_{i}<\left(U_{i}^{A c c}-L_{i}^{A c c}\right)$ are identified.

According to IFO technique considering membership and non-membership function for MOSOP (1)

$$
\begin{aligned}
& \mu_{W T(A)}(W T(A)) \\
& =\left\{\begin{array}{c}
1 \quad \text { if } W T(A) \leq L_{W T}^{A c c} \\
\frac{e^{-T\left(\frac{W T(A)-L_{W T}^{A c c}}{U_{W T}^{A c c}-L_{W T}^{A c c}}\right)}-e^{-T}}{1-e^{-T}} \text { if } \quad L_{W T}^{A c c} \leq W T(A) \leq U_{W T}^{A c c} \\
0 \quad \text { if } \quad W T(A) \geq U_{W T}^{A c c}
\end{array}\right. \\
& v_{W T(A)}(W T(A))=\left\{\begin{array}{c}
0 \quad \text { if } W T(A) \leq L_{W T}^{\mathrm{Re} j} \\
\left(\frac{W T(A)-L_{W T}^{\mathrm{Re} j}}{U_{W T}^{\mathrm{Re} j}-L_{W T}^{\mathrm{Re} j}}\right)^{2} \text { if } \quad L_{W T}^{\mathrm{Re} j} \leq W T(A) \leq U_{W T}^{\mathrm{Re} j} \\
1 \quad \text { if } \quad W T(A) \geq U_{W T}^{\mathrm{Re} j}
\end{array}\right.
\end{aligned}
$$

and

$$
\mu_{\delta(A)}(\delta(A))=\left\{\begin{array}{ccc}
1 & \text { if } \delta(A) \leq L_{\delta}^{A c c} \\
\frac{e^{-T\left(\frac{\delta(A)-L_{\delta}^{A c c}}{U_{\delta}^{A c c}-L_{\delta}^{A c c}}\right)}-e^{-T}}{1-e^{-T}} & \text { if } & L_{\delta}^{A c c} \leq \delta(A) \leq U_{\delta}^{A c c} \\
0 & \text { if } & \delta(A) \geq U_{\delta}^{A c c}
\end{array}\right.
$$

$$
v_{\delta(A)}(\delta(A))=\left\{\begin{array}{ccc}
0 & \text { if } \delta(A) \leq L_{\delta}^{\operatorname{Re} j} \\
\left(\frac{\delta(A)-L_{\delta}^{\operatorname{Re} j}}{U_{\delta}^{\operatorname{Re} j}-L_{\delta}^{\operatorname{Re} j}}\right)^{2} & \text { if } L_{\delta}^{\operatorname{Re} j} \leq \delta(A) \leq U_{\delta}^{\operatorname{Re} j} \\
1 & \text { if } \quad \delta(A) \geq U_{\delta}^{\operatorname{Re} j}
\end{array}\right.
$$

After determining the different membership functions for each of the objective functions, one can adopt following three types of decisions

i) According to the extension of the weighted intuitionistic min-max operator the MONLP (2) can be formulated as

Maximize $(\alpha-\beta)$

$$
\begin{aligned}
& w_{1}\left(\mu_{W T}(W T(A))\right) \geq \alpha ; w_{2}\left(\mu_{\delta}(\delta(A))\right) \geq \alpha ; \\
& w_{1}\left(v_{W T}(W T(A))\right) \leq \beta ; w_{2}\left(v_{\delta}(\delta(A))\right) \leq \beta ; \\
& \sigma(A) \leq[\sigma] ; \\
& A^{\min } \leq A \leq A^{\max } ; \\
& w_{1} \geq 0, w_{2} \geq 0, w_{1}+w_{2}=1 ; w_{1}, w_{2} \in[0,1] \\
& 0 \leq \alpha+\beta \leq 1 ; \alpha \geq \beta ; \alpha, \beta \in[0,1]
\end{aligned}
$$

ii) According to extension of weighted Yagar operator with $\lambda=2$

$$
\begin{aligned}
& \operatorname{Max}\left\{0,1-\sqrt{w_{1}\left(1-\mu_{W T}(W T(A))\right)+w_{2}\left(1-\mu_{\delta}(\delta(A))\right)}\right\} \\
& \text { Minimize }\left\{1, \sqrt{w_{1} v_{W T}(W T(A))+w_{2} v_{\delta}(\delta(A))}\right\}
\end{aligned}
$$

such that

$$
\begin{aligned}
& 0 \leq \mu_{W T}(W T(A))+v_{W T}(W T(A)) \leq 1 ; \\
& 0 \leq \mu_{\delta}(\delta(A))+v_{\delta}(\delta(A)) \leq 1 ; \\
& \mu_{W T}(W T(A)) \geq v_{W T}(W T(A)) ; \\
& \mu_{\delta}(\delta(A)) \geq v_{\delta}(\delta(A)) ; \\
& \sigma(A) \leq[\sigma] ; \\
& A^{\min } \leq A \leq A^{\max } ; \\
& w_{1} \geq 0, w_{2} \geq 0, w_{1}+w_{2}=1 ; w_{1}, w_{2} \in[0,1]
\end{aligned}
$$

iii)According to extension of weighted Hamacher operator with $\lambda=1.5$

$$
\operatorname{Max} \frac{\left\{\mu_{W T}(W T(A))\right\}^{w_{1}}\left\{\mu_{\delta}(\delta(A))\right\}^{w_{2}}}{1.5-0.5\left[\left\{1-\mu_{W T}(W T(A))\right\}^{w_{1}}\left\{1-\mu_{\delta}(\delta(A))\right\}^{w_{2}}\right]}
$$


Minimize

$\frac{w_{1} v_{W T}(W T(A))+w_{2} v_{\delta}(\delta(A))-0.5\left[\left\{v_{W T}(W T(A))\right\}^{w_{1}}\left\{v_{\delta}(\delta(A))\right\}^{w_{2}}\right]}{1+0.5\left[\left\{v_{W T}(W T(A))\right\}^{w_{1}}\left\{v_{\delta}(\delta(A))\right\}^{w_{2}}\right]}$

subject to the same constraint as (6.i)

\section{NUMERICAL ILLUSTRATION}

A well-known three bar planar truss structure is considered .The design objective is to minimize weight of the structure $W T\left(A_{1}, A_{2}\right)$ and minimize the deflection $\delta\left(A_{1}, A_{2}\right)$ along $x-$ axis and $y$-axis at loading point of a statistically loaded three bar planar truss subject stress $(\sigma)$ constraints on each of the truss members.

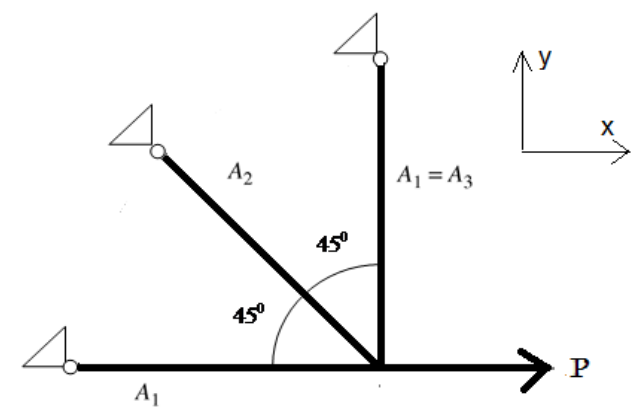

Fig .1. Design of three bar planar truss

The multi-objective optimization problem can be stated as follows

Minimize $W T\left(A_{1}, A_{2}\right)=\rho L\left(2 A_{1}+A_{2}\right)$

Minimize $\delta_{x}\left(A_{1}, A_{2}\right)=\frac{\rho L\left(2 A_{1}+A_{2}\right)}{E\left(2 A_{1}^{2}+2 A_{1} A_{2}\right)}$

Minimize $\delta_{y}\left(A_{1}, A_{2}\right)=\frac{\rho L A_{2}}{E\left(2 A_{1}^{2}+2 A_{1} A_{2}\right)}$

such that

$$
\begin{aligned}
& \sigma_{1}\left(A_{1}, A_{2}\right) \equiv \frac{P\left(2 A_{1}+A_{2}\right)}{\left(2 A_{1}^{2}+2 A_{1} A_{2}\right)} \leq\left[\sigma_{1}^{T}\right] \\
& \sigma_{2}\left(A_{1}, A_{2}\right) \equiv \frac{P}{\sqrt{2}\left(A_{1}+A_{2}\right)} \leq\left[\sigma_{2}^{T}\right] \\
& \sigma_{3}\left(A_{1}, A_{2}\right) \equiv \frac{\rho A_{2}}{\left(2 A_{1}^{2}+2 A_{1} A_{2}\right)} \leq\left[\sigma_{3}^{C}\right] \\
& A_{i}^{\min } \leq A_{i} \leq A_{i}^{\max } i=1,2 .
\end{aligned}
$$

Where $P=$ applied load ; $\rho=$ material density; $L=$ length ; $E=$ Young's modulus ; $A_{1}=$ cross sectional area of bar-1and bar-3. $A_{2}=$ cross sectional area of bar-2. $\delta_{x}$ and $\delta_{y}$ are the deflection of loaded joint along $x$ and $y$ axes respectively. $\left[\sigma_{1}^{T}\right]$ and $\left[\sigma_{2}^{T}\right]$ are the maximum allowable tensile stress for bar-1 and bar-2 respectively. $\left[\sigma_{3}^{C}\right]$ is maximum allowable compressive stress for bar -3 .

The optimal results of model (10) using t-norms and tconorms are shown in table 2 to 5 and Pareto optimal solution is shown in table 6 .

In table 2, for equal importance, the extension of weighted parameterized Hamecher-t-norm t-co-norm operator gives minimum structural weights as well as minimum deflection.

In table 3, for more importance on structural weight, the extension of weighted parameterized Hamacher-t-norm t-conorm operator gives minimum structural weights as well as minimum deflection.

In table 4 , for more importance on deflection, the extension of weighted parameterized Hamacher t-norm t-co-norm operator gives minimum structural weights where as weighted parameterized min-max t-norm t-co-norm operator gives minimum deflection.

In table 5, For more importance on deflection, the extension of weighted parameterized Hamacher-t-norm t-co-norm

\begin{tabular}{|c|c|c|c|c|c|c|c|}
\hline $\begin{array}{l}\text { Applied } \\
\text { Load } \\
\mathrm{P}(\mathrm{KN})\end{array}$ & $\begin{array}{l}\text { Material } \\
\text { Density } \\
(\rho)\left(\mathrm{KN} / \mathrm{m}^{3}\right)\end{array}$ & $\begin{array}{l}\text { Length } \\
\mathrm{L}(\mathrm{m})\end{array}$ & $\begin{array}{c}\text { Maximum } \\
\text { allowable } \\
\text { tensile } \\
\text { stress } \\
{\left[\begin{array}{c}\left.\sigma_{1}^{T}\right] \\
\left(\mathrm{KN} / \mathrm{m}^{2}\right)\end{array}\right.}\end{array}$ & $\begin{array}{c}\text { Maximum } \\
\text { allowable } \\
\text { tensile } \\
\text { stress } \\
{\left[\sigma_{2}^{T}\right]} \\
\left(\mathrm{KN} / \mathrm{m}^{2}\right)\end{array}$ & $\begin{array}{c}\text { Maximum } \\
\text { allowable } \\
\text { compressive } \\
\text { stress } \\
{\left[\sigma_{3}^{C}\right]} \\
\left(\mathrm{KN} / \mathrm{m}^{2}\right)\end{array}$ & $\begin{array}{c}\text { Young's } \\
\text { Modulus } \\
\text { E } \\
\left(\mathrm{KN} / \mathrm{m}^{2}\right)\end{array}$ & $\begin{array}{c}A_{i}^{\min } \text { and } A_{i}^{\max } \\
\text { of cross section of } \\
\text { bars } \\
10^{-4}\left(\mathrm{~m}^{2}\right)\end{array}$ \\
\hline 20 & 100 & 1 & 20 & 20 & 10 & $2 \times 10^{8}$ & $\begin{array}{l}A_{i}^{\min }=0.1, \\
A_{i}^{\max }=5, i=1,2\end{array}$ \\
\hline
\end{tabular}
operator gives minimum structural weights and minimum deflection.

Table.1 The input data for MOSOP (10) 
Table 2.Optimal weight for equal importance on structural weight and deflection i.e $w_{1}=w_{2}=w_{3}=1 / 3$ and for $\varepsilon_{W T}=1.28$

$$
\varepsilon_{\delta_{x}}=1.7 \quad \varepsilon_{\delta_{y}}=0.48
$$

\begin{tabular}{|c|c|c|c|c|c|}
\hline $\begin{array}{c}\text { Weighted } \\
\text { paremeterized t- } \\
\text { norm,t-conorm } \\
\text { operator }\end{array}$ & $A_{1}^{*} \times 10^{-4} \mathrm{~m}^{2}$ & $A_{2}^{*} \times 10^{-4} \mathrm{~m}^{2}$ & $W T^{*} \times 10^{2} \mathrm{KN}$ & $\delta_{x}^{*} \times 10^{-7} \mathrm{~m}$ & $\delta_{y}^{*} \times 10^{-7} \mathrm{~m}$ \\
\hline Min-max operator & 0.5495064 & 5 & 6.099013 & 1 & 0.8198038 \\
\hline Yager & 0.6060065 & 2.252335 & 3.464348 & 1 & 0.6501473 \\
\hline Hamacher & 0.6062547 & 2.246576 & 3.459086 & 1 & 0.6494715 \\
\hline
\end{tabular}

Table 3.Optimal weight for more importance on structural weight $w_{1}=0.6, w_{2}=0.2, w_{3}=0.2$ and for $\varepsilon_{W T}=1.28 \varepsilon_{\delta_{x}}=1.7$

$$
\varepsilon_{\delta_{y}}=0.48
$$

\begin{tabular}{|c|c|c|c|c|c|}
\hline $\begin{array}{c}\text { Weighted paremeterized } \\
\text { t-norm,t-conorm operator }\end{array}$ & $A_{1}^{*} \times 10^{-4} \mathrm{~m}^{2}$ & $A_{2}^{*} \times 10^{-4} \mathrm{~m}^{2}$ & $W T^{*} \times 10^{2} \mathrm{KN}$ & $\delta_{x}^{*} \times 10^{-7} \mathrm{~m}$ & $\delta_{y}^{*} \times 10^{-7} \mathrm{~m}$ \\
\hline Min-max operator & 0.5495096 & 5 & 6.099019 & 1 & 0.8198038 \\
\hline Yager & 0.6060065 & 2.252335 & 3.464348 & 1 & 0.6501473 \\
\hline Hamacher & 0.6062547 & 2.246576 & 3.459086 & 1 & 0.6494715 \\
\hline
\end{tabular}

Table 4.Optimal weight for more importance on deflection $w_{1}=0.2, w_{2}=0.6, w_{3}=0.2$ and for $\varepsilon_{W T}=1.28 \varepsilon_{\delta_{x}}=1.7 \quad \varepsilon_{\delta_{y}}=0.48$

\begin{tabular}{|c|c|c|c|c|c|}
\hline $\begin{array}{c}\text { Weighted paremeterized } \\
\text { t-norm,t-conorm operator }\end{array}$ & $A_{1}^{*} \times 10^{-4} \mathrm{~m}^{2}$ & $A_{2}^{*} \times 10^{-4} \mathrm{~m}^{2}$ & $W T^{*} \times 10^{2} \mathrm{KN}$ & $\delta_{x}^{*} \times 10^{-7} \mathrm{~m}$ & $\delta_{y}^{*} \times 10^{-7} \mathrm{~m}$ \\
\hline Min-max operator & 3.324846 & 3.905162 & 10.55485 & 0.2195391 & 0.08122667 \\
\hline Yager & 0.6060065 & 2.252335 & 3.464348 & 1 & 0.6501473 \\
\hline Hamecher & 0.6062547 & 2.246576 & 3.459086 & 1 & 0.6494715 \\
\hline
\end{tabular}

Table 5.Optimal weight for more importance on deflection $w_{1}=0.2, w_{2}=0.2, w_{3}=0.6$ and for $\varepsilon_{W T}=1.28 \quad \varepsilon_{\delta_{x}}=1.7 \quad \varepsilon_{\delta_{y}}=0.48$

\begin{tabular}{|c|c|c|c|c|c|}
\hline $\begin{array}{c}\text { Weighted paremeterized } \\
\text { t-norm,t-conorm operator }\end{array}$ & $A_{1}^{*} \times 10^{-4} \mathrm{~m}^{2}$ & $A_{2}^{*} \times 10^{-4} \mathrm{~m}^{2}$ & $W T^{*} \times 10^{2} \mathrm{KN}$ & $\delta_{x}^{*} \times 10^{-7} \mathrm{~m}$ & $\delta_{y}^{*} \times 10^{-7} \mathrm{~m}$ \\
\hline Min-max operator & 0.5495098 & 5 & 6.099020 & 1 & 1.8198039 \\
\hline Yager & 0.6060065 & 2.252335 & 3.464348 & 1 & 0.6501473 \\
\hline Hamacher & 0.6062547 & 2.246576 & 3.459086 & 1 & 0.6494715 \\
\hline
\end{tabular}

Table 6. Pareto Optimality test for $w_{1}=w_{2}=w_{3}=1 / 3$

\begin{tabular}{|c|c|c|c|c|c|}
\hline$R$ & $A_{1}^{*} \times 10^{-4} m^{2}$ & $A_{2}^{*} \times 10^{-4} m^{2}$ & $W T^{*} \times 10^{2} \mathrm{KN}$ & $\delta_{x}^{*} \times 10^{-7} \mathrm{~m}$ & $\delta_{y}^{*} \times 10^{-7} \mathrm{~m}$ \\
\hline 0.1 & 0.6062548 & 2.246580 & 3.459090 & 1 & 0.6494715 \\
\hline
\end{tabular}

\section{CONCLUSIONS}

In this paper, we have proposed a method to solve multiobjective structural model in intuitionistic fuzzy environment. Here binary t-norms are expressed in extended n-ary t-norms and discussed their basic properties and some special cases. The said model is solved by using t-norms and t-conorm based on intuitionistic fuzzy optimization technique. A main advantage of the proposed method is that it allows the user to concentrate on the actual limitations in a problem during the specification of the flexible objectives. This approximation method can be applied to optimize different models in various fields of engineering and sciences.

\section{ACKNOWLEDGMENTS}

Authors would like to thank referees for their helpful comments.

\section{REFERENCES}

[1] Angelov,P.P.1993. Optimization in intuitionistic fuzzy environment. Fuzzy Sets and Systems 86, 299-306.

[2] Dey,S and Roy,T.K.2014. Optimized solution of two bar truss design using intuitionistic fuzzy optimization technique International Journal of Information Engineering and Electronic Business,2014(3), 45-51.

[3] Atanassov,K.1986.Intuitionistic fuzzy sets, Fuzzy sets and Systems, 20,87-96.

[4] Zadeh,L.A.1965.Fuzzy set, Information and Control, 8(3),338-353. 
[5] Yager,R.R.1978.Fuzzy decision making including unequal objectives Fuzzy Sets and Systems,1,87-95.

[6] Alsina,C.,Trillas,C and Valverde,I.1981. On some logical connectives for fuzzy set theory.J.Math.Anal.Appl.93,1526.

[7] Deschrijver, G., Cornelis, C.and Kerre, K.K., 2004. On the representation of intuitionistic fuzzy t-norms and tconorms IEEE Trans.Fuzzy Syst,12,45-61.

[8] Xeshui.Xu.2007.Intuitionistic Fuzzy Aggregation Operator, IEEE Trans .Fuzzy Syst,15(6),1179-1187.
[9] Dey,S and Roy,T.K.2015.Multi-Objective Structural Optimization using Fuzzy and Intuitionistic Fuzzy Optimization Technique. International Journal of Intelligent Systems and Applications,5,57-65.

[10] Dey,S and Roy,T.K.2016. Multi-objective Structural Design Problem Optimization using Parameterized tnorm based on Fuzzy optimization Programming Technique. Journal of Intelligence and fuzzy systems,30(2),971-982.

[11] Zimmermann,H.J. 1978. Fuzzy programming and linear programming with several objective functions. Fuzzy sets and systems, 1(1), 45-55. 